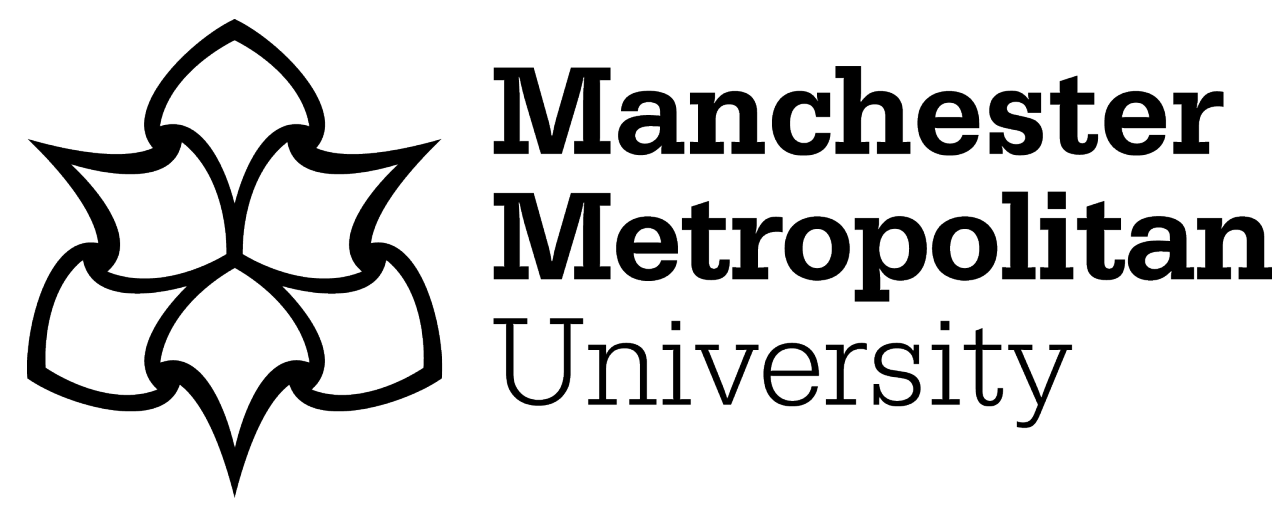

Chang, YH, Wang, W ORCID logoORCID: https://orcid.org/0000-0002-12254011, Chang, JY and Mottershead, JE (2019) Compressed sensing for OMA using full-field vibration images. Mechanical Systems and Signal Processing, 129. pp. 394-406. ISSN 0888-3270

Downloaded from: https://e-space.mmu.ac.uk/622890/

Version: Accepted Version

Publisher: Elsevier

DOI: https://doi.org/10.1016/j.ymssp.2019.04.031

Usage rights: Creative Commons: Attribution-Noncommercial-No Derivative Works 4.0

Please cite the published version 


\title{
Compressed Sensing for OMA using Full-Field Vibration Images
}

\author{
Yen-Hao Chang ${ }^{1,2}$, Weizhuo Wang ${ }^{3}$, Jen-Yuan Chang ${ }^{2}$ and John E Mottershead ${ }^{1}$ \\ ${ }^{1}$ Department of Mechanical, Aerospace and Materials Engineering, \\ University of Liverpool, UK. \\ ${ }^{2}$ Department of Power Mechanical Engineering, National Tsing Hua University, \\ Taiwan. \\ ${ }^{3}$ School of Engineering, Manchester Metropolitan University, UK.
}

\begin{abstract}
:
Optically-acquired data, typically from digital image correlation, is increasingly being used in the area of structural dynamics, particularly modal testing and damage identification. One of the problems with such data is its extremely large size. Single images regularly extend to tens or even hundreds of thousands of data points and many thousands of images may be required for a vibration test. Such data must be stored and transmitted efficiently for later remote reconstruction and analysis, typically operational modal analysis. It is this requirement that is addressed in the research presented in this paper. This research builds upon previous work whereby digitised optical data was projected onto an orthogonal basis with coefficients (shape descriptors) of either greater or lesser significance; those deemed to be insignificant, according to a chosen threshold being removed. Data reduction by a combination of shapedescriptor decomposition and compressed-sensing is applied to an industrial printed circuit board and reconstructed for operational modal analysis by $\ell_{1}$ optimisation.
\end{abstract}

Keywords: Compressed sensing, shape descriptor, sparse representation, structural health monitoring, operational modal analysis.

\section{Introduction.}

In recent years the demand for online monitoring and remote inspection has brought about a huge surge of research activity in structural health monitoring (SHM). Applications range from the preservation of historical building [1] to the monitoring and maintenance of offshore wind turbines [2] with the purpose of miminising the costs of restoration, deterioration, machine down-time etc. Wireless sensor networks are increasingly prevalent. Hierarchical decentralized SHM as presented by Hackmann et al. [3] was said to be capable of only activating sensors in damaged regions and keeping others idle. Other than conventional accelerometer or strain gauge measurement, non-destructive acoustic [4] and non-contact vision-based [5,6] measuring methods are also being adopted. Yang et al. [7] proposed a highfidelity simulation and visualisation method for full-field dynamic structural responses based on phase-based motion magnification (Wadhwa et al. [8]) as an alternative to finite element analysis. Along with the expanding application of video-based monitoring, image transmission or storage plays an important role in realising the implementation of state-of-art techniques in industry. Yang and Nagarajaiah [9] suggested that images gathered from measurement can be first compressed for transmission and afterwards recovered by using the compressed sensing 
(CS) technique developed by Donoho [10]. As explained in [9], the main factor in industrial adoption of SHM research is the efficient and reliable transfer and processing of data. A discussion on closing the gap between academic research and industrial deployment was presented by Cawley [11] and a review of signal processing, as applied to SHM, was provided by Amezquita-Sanchez and Adeli [12].

Digital image correlation (DIC) is presently the most widely applied computer vision method for strain analysis, and its application in dynamic measurement is becoming regular with promising new techniques breaking or circumventing the limitations related to stereo vision, high sampling rate and restricted line-of-sight. Yu and Pan [13] presented a colour stereo-digital image correlation method using just one 3CCD colour camera without trade-off of spatial resolution and inaccessibility by the arrangement of mirrors and colour filters for the separation of light beams into its individual colour components. Yu and Pan [14] considered the combination of a four-mirror adapter with a single high-speed camera and validated the proposed system by comparison with finite element analysis. Later, the concepts introduced in [13] and [14] were integrated, i.e. single high-speed camera with mirror adapter and colour filters [15], and two experiments (shape measurement of a curved surface and vibration measurement of a Chinese double-side drum) were carried out for validation purposes. Instead of two industrial cameras, Chi et al. [16] used a consumer-grade Single Lens Reflex camera with a four-mirror adapter for the acquisition of images, and the low-cost and portable system was demonstrated on thermal deformation measurement of an alumina ceramic plate and a stainless-steel plate subjected to radiation heating. Under a broadband white-noise excitation, high-frequency modes have relative low amplitudes compared to low-frequency modes, and the measurement of corresponding displacement images by DIC then becomes quite challenging. Molina-Viedma et al. [17] used phase-based video motion processing technique for the magnification of displacement images of low-amplitude high-frequency modes together with stroboscopic sampling to overcome the high sampling rate required by the NyquistShannon theorem - the highest frequency of an identified mode was $6710 \mathrm{~Hz}$ without the necessity of high-level excitation. One of the disadvantages of applying DIC systems is that the accuracy of displacement estimates is limited by the surface curvature of the specimen most demonstrations are carried out on plate-like structures. However, Patil et al. [18] provided a method similar to those for image stitching, based on common reference points for the merging of mode shapes of sub-areas of large or curved structures, such as on motor-vehicle tyres or large turbine blades, to acquire the full mode shape of the test article.

Although the world seems to be full of diversity, redundancy is ubiquitous in nature, for example sound waves and electromagnetic waves can be represented by only a few frequency components corresponding to particular tones or colours, and the shape or pattern of astronomical objects, creatures, or even bacteria and viruses are of specific geometries, meaning that they are relatively sparse compared to the diversity of possibility that might be artificially created were science sufficiently advanced. Candès et al. [19] and then Donoho [10] proposed to take advantage of this property of sparsity to recover a signal from a number of frequency-domain components (or measurements) that was small when compared to the dimension of the original signal. The concept is widely applied today in several disciplines, and numerous examples can be seen in recent publications. Magnetic resonance imaging (MRI) has particularly benefitted from the application of CS [20] resulting in significantly improved scan times [21, 22] and the removal of artifacts and reconstruction errors [23]. Arridge et al. [24] applied CS to photoacoustic tomography imaging of living tissue and showed similar 
improvements in acquisition speed with good spatial resolution and contrast. Gangopadhyay et al. [25] highlighted the efficacy of CS for ultra-low power wireless and wearable biosensors, and Craven et al. [25] identified a reduction in healthcare costs in ambulatory monitoring of bioelectric signals. CS has been applied in such diverse fields as Solar Flare X-Ray Observation [27] and super resolution imaging in atomic force microscopy [28].

In the field of engineering dynamics, and with CS sensors still at the prototype stage, Yang and Nagarajaiah [29] explored the potential of combining output-only blind source separation (BSS) and CS. Qin et al. [30] developed a sparse component analysis (SCA) for the detection of single source points (SSPs) with sparsity in the time-frequency domain for OMA. Park et al. [31] considered the application of CS to modal testing in the presence of close modes. They showed that the free vibration displacement response could be decomposed in terms of its singular values (SVD) where the left singular vectors are understood to be estimates of the system mode shapes. Uniform- and random-time sampling were considered, as was randommatrix selection (Gaussian) from uniform samples. The latter was applied to a physical bridge structure with reconstruction using $\ell_{1}$ optimisation.

The shape descriptor (SD) technique is founded upon similar understanding to that of CS, i.e. images have spatial redundancy, and the spatial patterns can be effectively represented by linear combinations of suitable basis functions and coefficients. The earliest work on SDs by $\mathrm{Hu}$ [35] used geometric moments, with invariance of rotation, translation and scaling, for image analysis and pattern recognition. The first suggestion that SDs might be useful in the interpretation of full-field images of displacement or vibration of structures, typically obtained by DIC, was made by Wang et al. [32,33]. The objective was to project the image onto a basis of orthonormal kernel functions, such that those basis terms with large coefficients were retained while the others, with coefficients deemed to be insignificant, were discarded. Criteria based on the fidelity of the reconstructed image were developed in order to determine the number of retained kernels. Initial studies made use of classical polynomials, such as Zernike and Tchebichef (Chebyshev), which are found to be orthogonal over circular or rectangular fields respectively. Then by applying SD image reduction to both measured full-field data and finite element (FE) outputs, model updating was carried out by converging the FE model upon the test data by minimisation of the SD residual [34, 35]. Engineering structures are mostly neither circular nor rectangular in shape and it then became necessary to apply generally adhoc modification to the kernels before orthogonalising them again by the Gram-Schmidt method [35]. This led to the development of a general procedure applicable to full-field data from engineering structures of arbitrary shape, known as the adaptive geometric moment descriptor (AGMD) [36]. The advantages of this approach were exemplified in the vibration study of a Fiat Panda bonnet liner, having a complicated curved surface and holes, with fullfield modal testing carried out in the SD (AGMD) domain - rather than in the physical domain of a limited number of acceleration sensors. Recent research by Chang et al. [37] addresses the fact that the pre-defined AGMD, though general, is not necessarily the most efficient (or compact) basis. They presented an algorithm inspired by K-SVD which uses measurements to directly update the chosen kernel functions to obtain a more representative and concise reconstruction of full-field data.

A combination of SD and CS techniques is proposed in the paper with the aim of developing a much more compact compression in both the time signal and the spatial pattern of full-field dynamic displacement images measured by high-speed 3D DIC systems for data transmission and storage. A brief review of the SD method is given in $\S 2$, while in $\S 3$ a 
mathematical description of CS is provided. An industrial case study in the form of a partiallyobserved printed circuit board is presented in $\S 4$ and the combined SD/CS procedure is explained in $\S 5$. In $\S 6$ CS is applied to raw data from a single circuit-board DIC image (i.e. without SD decomposition). This is shown to be an expensive computation, which justifies the development of the combined SD/CS approach demonstrated experimentally in §8, including OMA, carried out on CS-recovered data from SD signals and kernel functions.

\section{Review of the Shape-Descriptor Method}

The SD method is based on the projection of an image (displacement, strain, vibration mode etc.) onto a basis of orthogonal polynomials. Classical polynomials, typically Zernike and Tchebichef, and AGMD kernels are all preselected independently of the image in question and therefore do not, in general, provide the most economical reduction. The present authors developed a basis updating procedure [37] inspired by the K-SVD algorithm [38].

The basis of $v \leq n$ kernel functions may be written as,

$$
\mathbf{A}_{k}=\left[\begin{array}{lllll}
\mathbf{a}_{1} & \cdots & \mathbf{a}_{w} & \cdots & \mathbf{a}_{v}
\end{array}\right]_{k}
$$

with SD coefficients,

$$
\mathbf{S}_{k}=\left[\begin{array}{c}
\mathbf{s}_{1}^{T} \\
\vdots \\
\mathbf{s}_{w}^{T} \\
\vdots \\
\mathbf{s}_{v}^{T}
\end{array}\right], \quad \mathbf{s}_{w}^{T}=\left(\begin{array}{llll}
s_{w 1} & S_{w 2} & \cdots & s_{w t}
\end{array}\right)
$$

where $t$ denotes the number of time-sequenced vibration DIC images and $n$ is the number of DIC data points present in each image. The procedure advances iteratively by determining at each $\operatorname{step}(k=1,2, \ldots)$ the complement of the sum of the kernel-function contributions to the data $\mathbf{X}$, with the $r^{\text {th }}$ term missing,

$$
\mathbf{X}_{r}=\mathbf{X}-\sum_{\substack{w=1 \\ w \neq r}}^{v} \mathbf{a}_{w} \mathbf{s}_{w}^{T} ; \quad \mathbf{X}, \mathbf{X}_{r} \in \mathrm{R}^{n \times t} ; \quad r=1,2, \ldots, v
$$

Singular value decomposition (SVD) is then applied such that,

$$
\mathbf{X}_{r}=\mathbf{U} \Sigma \mathbf{V} ; \quad \mathbf{U}=\left[\begin{array}{llll}
\mathbf{u}_{1} & \mathbf{u}_{2} & \cdots & \mathbf{u}_{n}
\end{array}\right]
$$

with the $r^{\text {th }}$ column of $\mathbf{A}_{k}$ updated such that,

$$
\mathbf{a}_{r}=\mathbf{u}_{1}
$$

Upon completion, when all the kernels have been updated, Gram-Schmidt orthonormalisation is applied and any repeated kernels are removed. A new set of coefficients are then produced by projecting the image onto the new basis functions. Iteration precedes until the difference between the data and its SD representation falls below a chosen threshold,

$$
\left\|\mathbf{X}-\mathbf{A}_{k} \mathbf{S}_{k}\right\|_{F} \leq \varepsilon
$$

At each iteration any SD coefficients and kernels deemed to be insignificant are removed. Finally, a set of kernels and SDs, based on the data and generally much smaller in dimension than the original AGMD system, are produced. 


\section{Compressed Sensing Method.}

CS is applicable to both signals (in time) and images (in 2-D or 3-D space) but is perhaps easiest explained in terms of an $\mathrm{N}$-vector of time-varying signals. Conventional signal processing using the Nyquist-Shannon sampling theory requires that the sampling rate shall be at least twice the highest frequency present in the signal. CS overturns this theory using principles of sparsity, incoherence and $\ell_{1}$ optimisation.

Consider the decomposition of the signal vector as,

$$
\mathbf{X}=\Psi \mathbf{u}=\sum_{j=1}^{N} \Psi_{j} u_{j} ; \quad \Psi \in \square^{N \times N}\left(\text { or } \square^{N \times N}\right)
$$

where $\left\{\psi_{j}\right\}_{j=1}^{N}$ is an orthonormal basis and the solution of coefficients $u_{j}$ is generally an underdetermined problem with an infinity of solutions. If the system is $k$-sparse, it means that there are $k$ significant coefficients and another $N-k$ coefficients which are considered small enough to be neglected. One problem with this is that $k$ is usually unknown without first determining all the coefficients and then deciding which ones should be retained - this is the adaptive approach (as applied in the SD method). An important aspect of CS is that it is non-adaptive and does not require the explicit solution of the underdetermined problem described in (7).

In CS [39], $\left\{\Psi_{j}\right\}_{j=1}^{N}$ is known as the sparsifying basis and should be minimally coherent with the so-called measurement basis,

$$
\mu(\Phi, \Psi)=\sqrt{N} \cdot \max _{1 \leq p, q \leq n}\left|\left\langle\boldsymbol{\varphi}_{q}, \boldsymbol{\Psi}_{p}\right\rangle\right|
$$

where the measurement is expressed as,

$$
\mathbf{y}=\boldsymbol{\Phi x} ; \quad \boldsymbol{\Phi}=\left[\begin{array}{c}
\boldsymbol{\varphi}_{1}^{T} \\
\boldsymbol{\varphi}_{2}^{T} \\
\vdots \\
\boldsymbol{\varphi}_{m}^{T}
\end{array}\right] \in \square^{m \times N}\left(\text { or } \square^{m \times N}\right)
$$

and $m$ denotes the number of measurements.

If the measurements are selected by the use of delta functions, then the product $\Phi \times \Psi$ results in simply the values of the sparsifying basis at those instants then the delta functions are applied. One advantage of using the delta-function measurement basis is that the coherence (8) is known to be minimal when $\Psi$ is the Fourier-transform basis. Thus, in the case of discrete time observation the Fourier basis in equation (7) is then defined as,

$$
\psi_{j}=F \mathbf{x}_{t}=\sum_{t=0}^{N-1} \mathbf{x}_{t} \mathrm{e}^{-\mathrm{i} \omega_{j} t} ; \quad \omega_{j}=\frac{2 \pi j}{N} ; \quad j=0,1, \ldots, N-1 ; \quad \mathrm{i}=\sqrt{-1}
$$

Of course, different measurement and sparsifying bases may be used and it is known that a random measurement basis tends to be incoherent with any orthonormal sparsifying basis. Thus, for efficient storage and transmission of signals or images it is sufficient to define an incoherent product $\Phi \times \Psi$ where the signal is sparse according to the basis $\Psi$ and the measurements (to be stored or transmitted) are compressed according to the basis $\Phi$. Reconstruction of the signal is said to be achievable with overwhelming probability by $\ell_{1}$ optimisation [39, 40] when, 


$$
m \geq b \times \mu^{2}(\boldsymbol{\Phi}, \Psi) k \log (n)
$$

where $b$ is a small positive constant. Then the estimated coefficients are given by,

$$
\hat{\mathbf{u}}=\arg \min _{\mathbf{u}}\|\mathbf{u}\|_{1} \quad \text { subject to } \Phi \Psi \mathbf{u}=\mathbf{y}
$$

and the reconstructed signal may be expressed as,

$$
\hat{\mathbf{x}}(t)=\boldsymbol{\Psi} \hat{\mathbf{u}}
$$

The advantage of $\ell_{1}$ minimisation is easily illustrated by the ball of unit norm in the space of the coefficients $u_{i}$. Figure 1 shows the simple and easily visualised 2-dimensional case. Whereas the 2-norm solution is defined by 2 coefficients, the 1-norm solution is of minimal dimension $k=1$. Thus, by the non-adaptive CS approach it is not necessary to determine the number $k$ of significant coefficients explicitly, since this is done as part of (and within) the $\ell_{1}$ minimisation procedure.

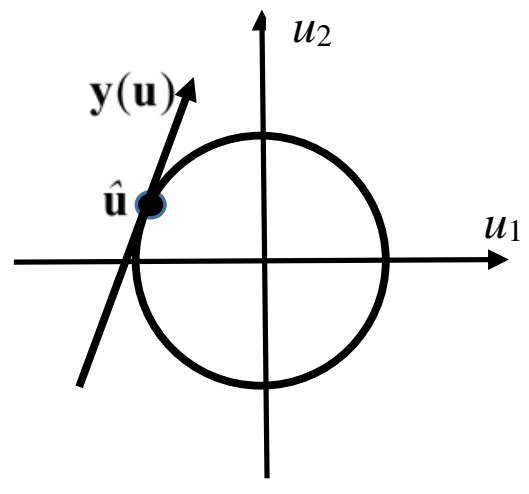

(a) $\ell_{2}$ norm

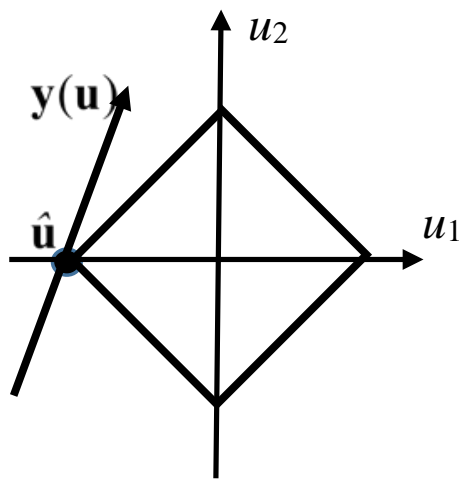

(b) $\ell_{1}$ norm

Fig. 1. $\ell_{2}$ and $\ell_{1}$ norm solutions

In the case of vibration images, as described in what follows, the Fourier Transform basis is used for sparsification and the measurements are delta functions. The minimum total variation (TV) algorithm with equality constraints is applied. This algorithm is fully described in Appendix E of [39] in which the gradients of the image, which tend to be sparser than displacements, are considered and are thus better suited to CS. The TV of $x$ is thus given by,

$$
\operatorname{TV}(x)=\sum_{p q} \sqrt{\left(D_{h ; p q} x\right)^{2}+\left(D_{v ; p q} x\right)^{2}}
$$

where the subscripts $h$ and $v$ denote horizontal and vertical,

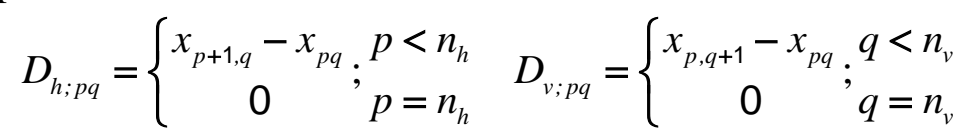

and $n_{h}$ and $n_{v}$ denote the number of data points in each of the two directions.

\section{Experimental Case Study}

The circuit board of overall dimensions $120 \times 240 \mathrm{~mm}^{2}$, is a real experimental example of DIC data from an industrial company. Measurements were made using a two-camera, 3-D DIC arrangement with a zero-mean normalised sum of squared differences (ZNSSD) criterion. The resolution of the recorded images was 512 pixels by 1280 pixels. The circuit board was partly obscured by other components so that the camera had only two separate parts of the circuit 
board in view, as shown in Fig. 2. The structure was excited randomly within the frequency range of 200 to $1000 \mathrm{~Hz}$, and the sampling frequency of the highspeed cameras was set at 2000 Hz. The total duration of measurement was $1.579 \mathrm{~s}$, and hence the resulting number of steps was 3158.

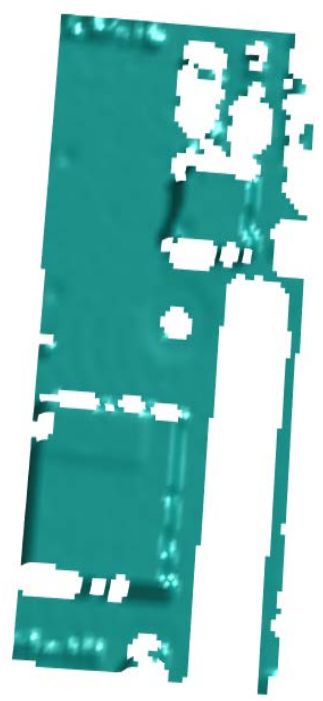

(a) Part 1

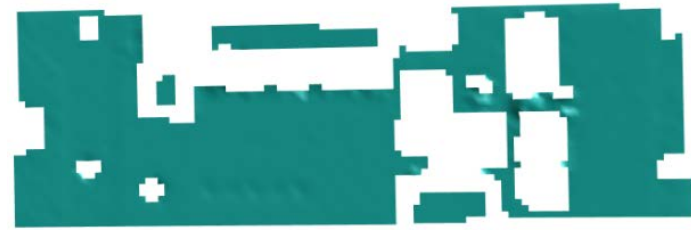

(b) Part 2

Fig. 2. Circuit board parts 1 (a) and 2 (b).

The printed circuit board forms the subject of tests carried out in subsequent sections to illustrate the application of SD and CS procedures to industrial full-field vibration test scenarios.

\section{Compressed Sensing Procedure}

Each of the 3158 DIC images consists of a total number of DIC data points of 8091 for the two parts of the circuit board - after removal of points corresponding to the circuit-board holes. There are two alternative approaches that might be considered here. First, the TV algorithm might be applied to the 3158 DIC images, and secondly CS might be applied to the 8091 data-points time signals. It will be demonstrated subsequently that the first approach incurs an unacceptable computational cost and compression achieved by the second approach is vastly inferior to the combined SD/CS method proposed herein.

Instead of either of above approaches CS will now be considered with SD decomposition applied a-priori. By this approach each of the 3158 images is projected onto a set of timeindependent orthogonal kernel functions, with coefficients, known as Shape Descriptors (SD), that form a time-varying signal. The kernels are developed from the data itself using an algorithm similar to K-SVD [38], which has the advantage of significantly reducing the number of kernels necessary to reproduce the original image. In this way, for maximum efficiency of storage and transmission, CS is applied both to the SD signals and to each of the kernel functions separately.

For CS of the SD signals, the measurement basis takes the form of delta functions, random in time [40]. The Fourier transform basis is applied for sparsification. The kernel function gradients require 2-D Fourier transformation for sparsification and the measurements are taken along equi-spaced radial scan lines taken from the centre of the image as illustrated in Fig. 3. 
The complete CS procedure is illustrated in Fig. 4. DIC data at the holes in the printed circuit board were replaced by zero values, which leads to discontinuities in the gradient field at the holes boundaries and some degradation in the sparsity of the measurement.

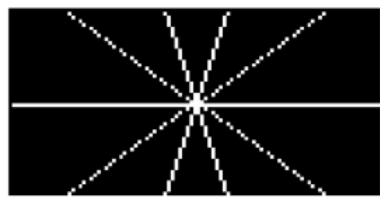

(a) pattern of 5 scan lines

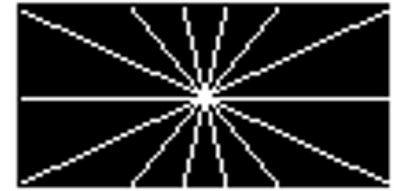

(c) pattern of 7 scan lines

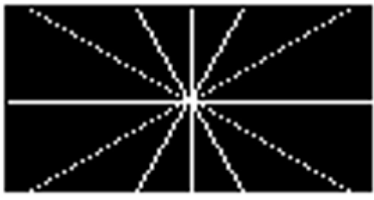

(b) pattern of 6 scan lines

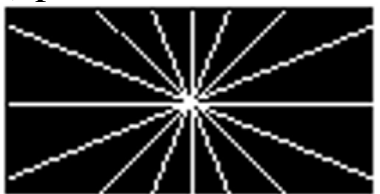

(d) pattern of 8 scan lines

Fig. 3. Patterns with different numbers of scan lines.

Reconstruction of SD signals and kernels by $\ell_{1}$ optimisation is described in $\S 2$ and illustrated in Fig. 5. The procedure described in Figures 4 and 5 allows for in-situ measurement and compression, with remote reconstruction of transmitted compressed data for OMA.

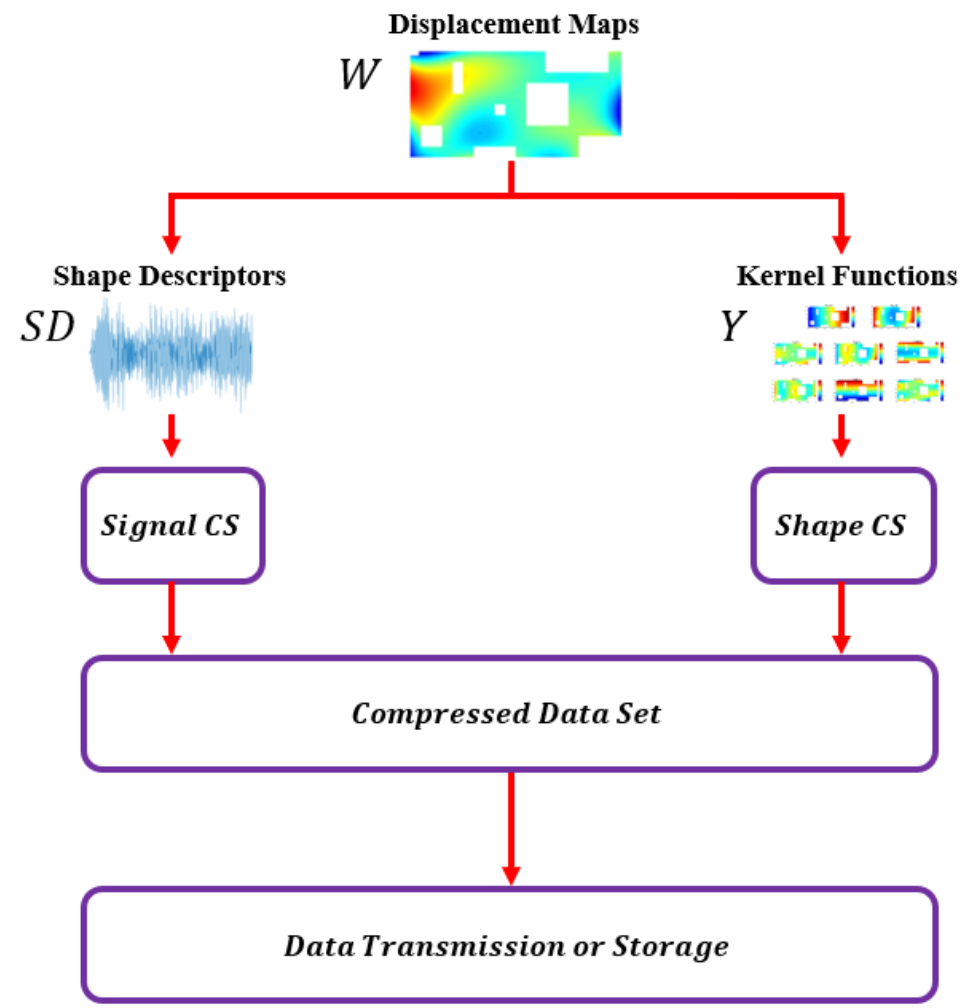

Fig. 4. Flow chart of the compression scheme. 


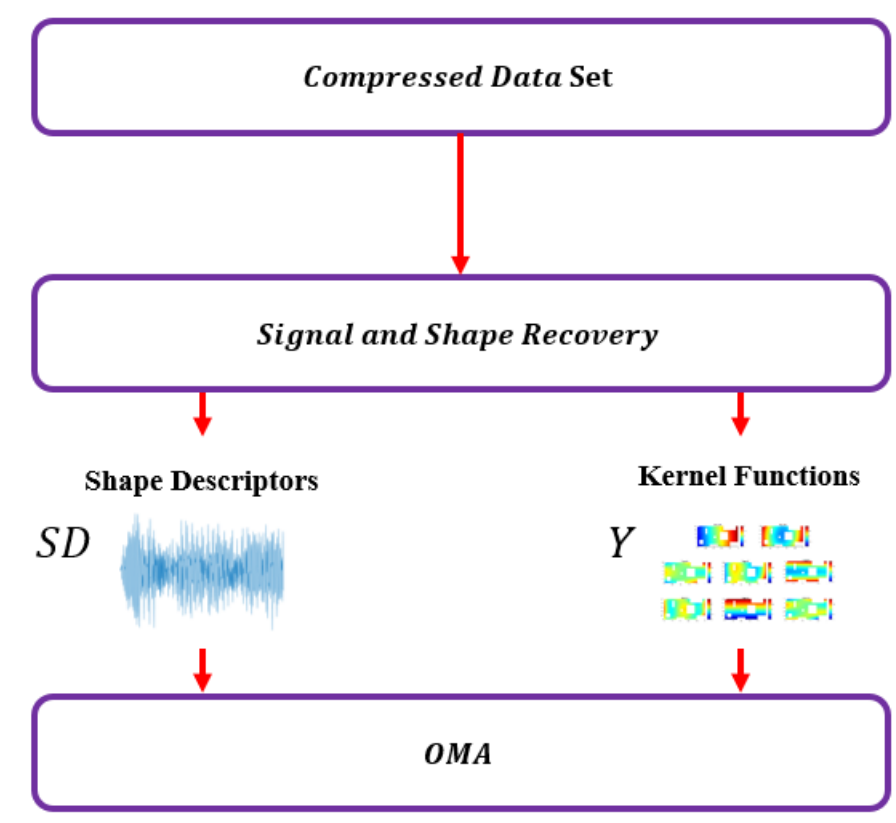

Fig. 5. Flow charts of the recovery scheme.

\section{Compressed Sensing of a Single Image}

In this section, the problem of applying CS directly a single DIC image is considered. This corresponds the first of two possible approaches postulated in $\S 4$, and the effect of applying different numbers of scan lines is investigated. Firstly, however, the correlation of CSrecovered images with time-progressing raw DIC data is shown in Fig. 6 using only nine scan lines. It is immediately apparent that the number of scan lines is not enough to obtain a sufficiently good correlation. The lowest and highest correlations are found to be 0.3546 and 0.8695 at time instants 3020 and 3029 respectively. The corresponding DIC displacement images are shown in Fig. 7.

The advantage of increased sparsity of data-point gradients over data-point values directly is illustrated in Fig. 8 for part 2 of the circuit board, where it is seen the number of negligibly small gradients is more than twenty times the number of small data-point values for the displacement image with the lowest correlation. The data-point gradients for the lowest and highest correlated images are compared in Fig. 9. Although the highest correlated image has the highest gradients it is the shape of the curves in the figure that determine the sparsity. The blue curve for the lowest correlated image bends over and becomes almost flat at around data point 2300 to 2700 . It contains a greater number of significant data-point gradients than the sharper red curve, which contains only a few significant gradients near the right-hand tip. The number of data points shown in Figs. 8 and 9 is the total remaining after eliminating those where there are holes in the circuit board material.

To improve the correlation, the number of scan lines should be increased. The effect of doing so is shown in Fig. 10, where the lowest correlated image is recovered almost perfectly when CS is applied with 25 scan lines. The recovered displacement images corresponding to the data points in Fig. 10 are illustrated in Fig. 11 for purposes of comparison. 


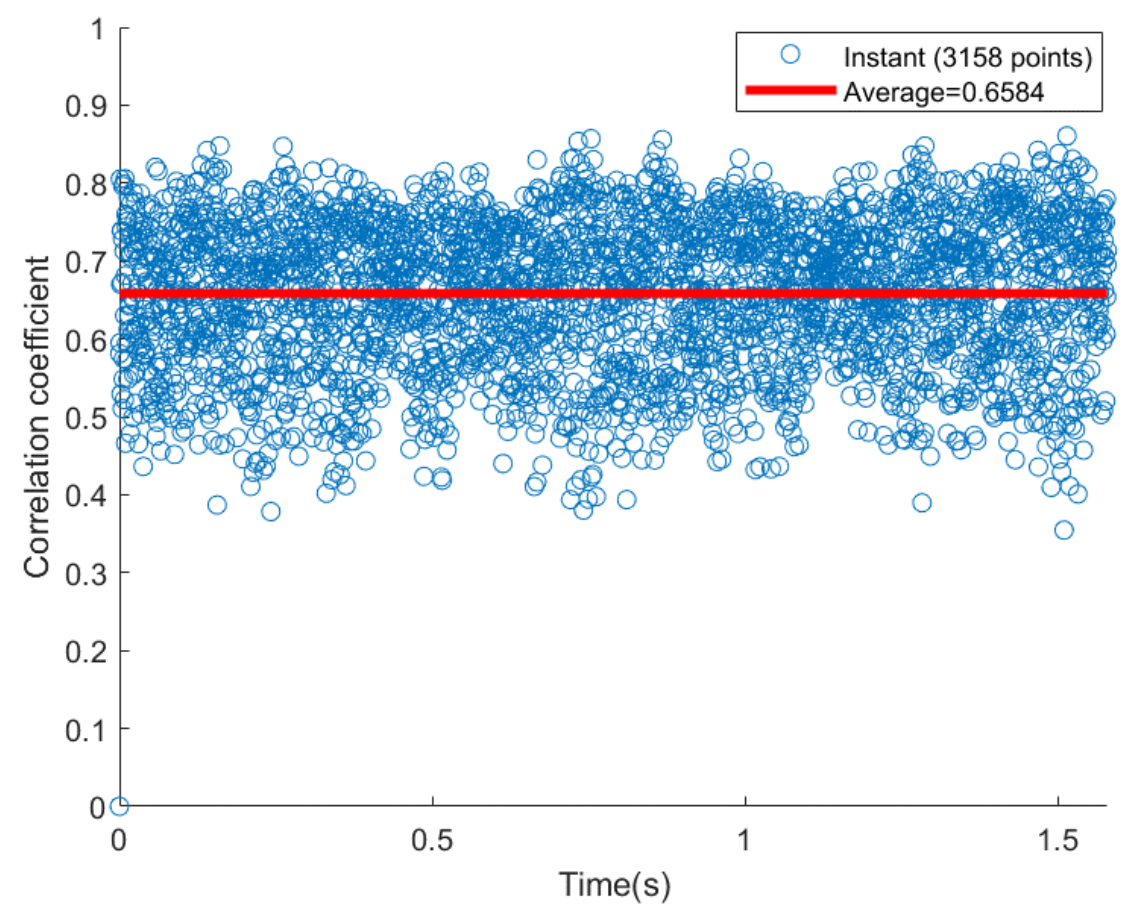

Fig. 6. Correlation coefficients of recovered displacement images: circuit board part2.

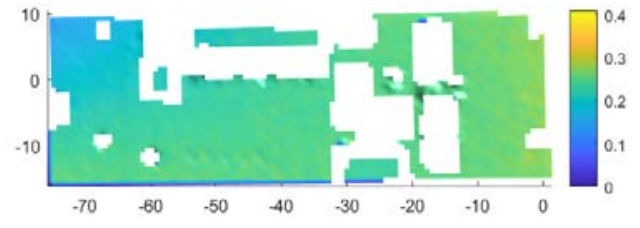

(a) Lowest

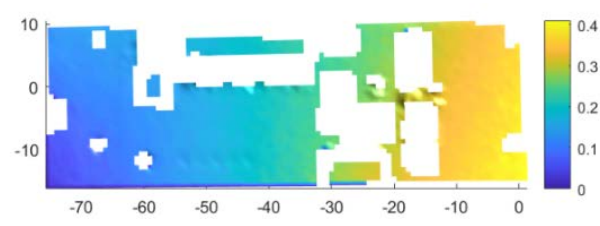

(b) Highest

Fig. 7. Displacement images (mm) with the lowest and highest correlation coefficients. 


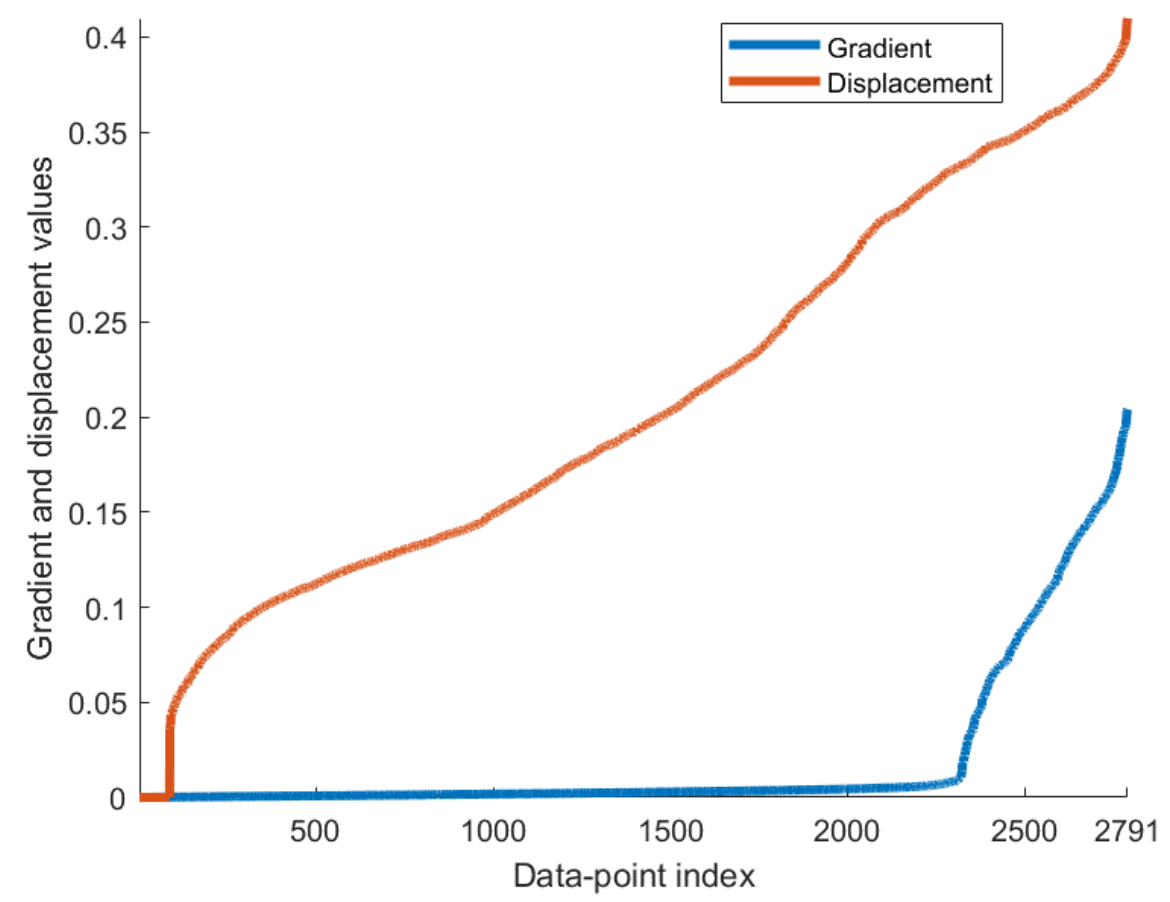

Fig. 8. Sorted absolute values of data-point gradient and displacement vs. data-point index: highest correlation coefficient.

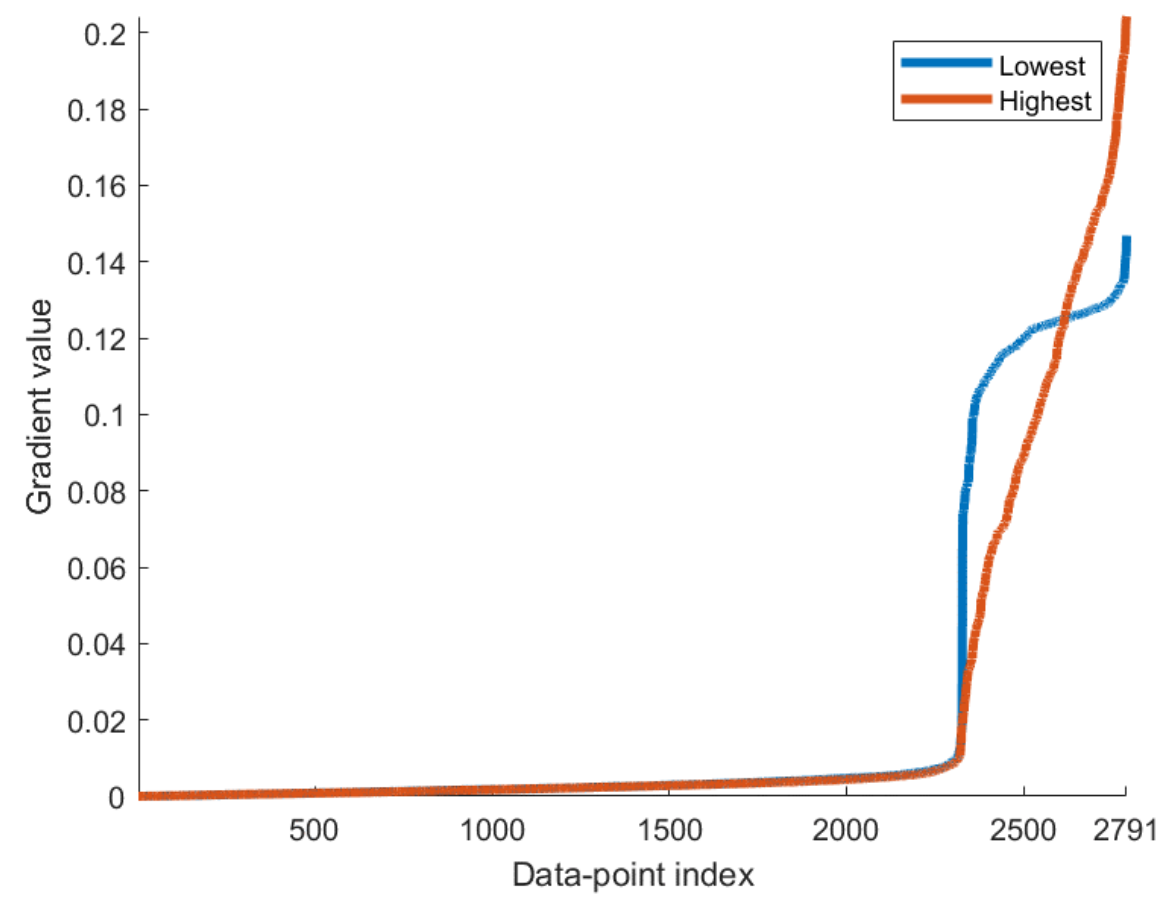

Fig. 9. Sorted absolute values of data-point gradients vs. data-point index: lowest and highest correlation coefficients. 


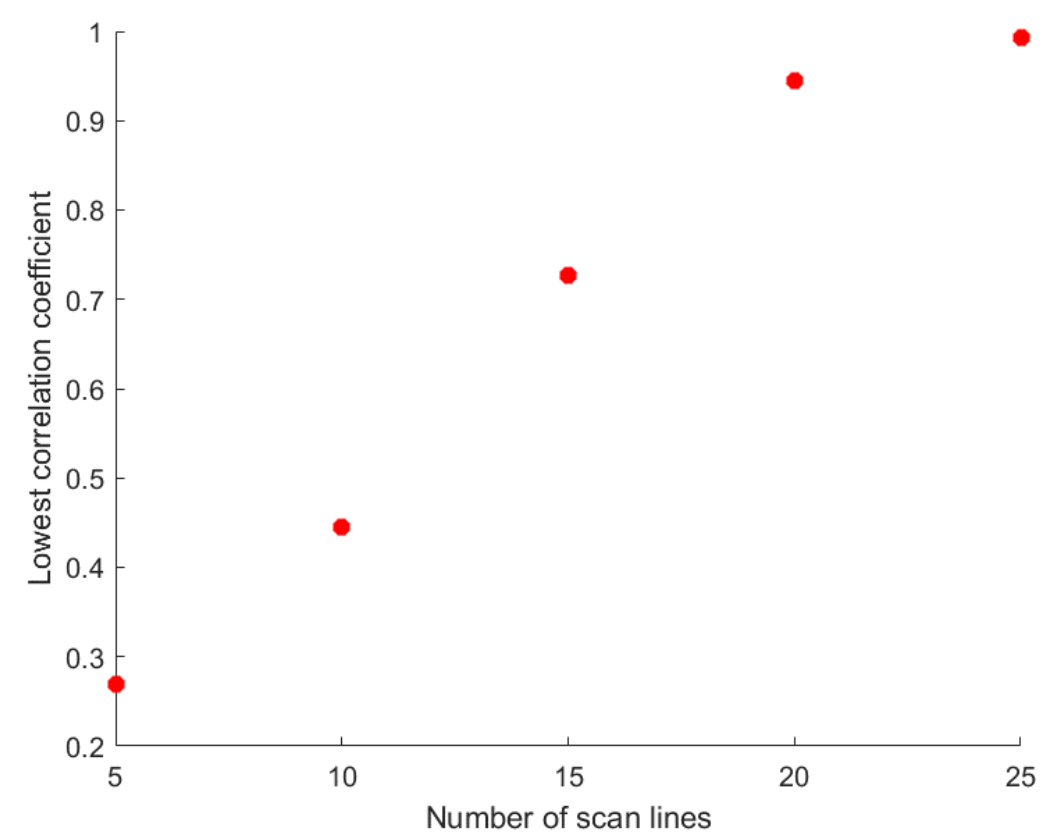

Fig. 10. Correlation coefficients of recovered images vs. number of scan lines.

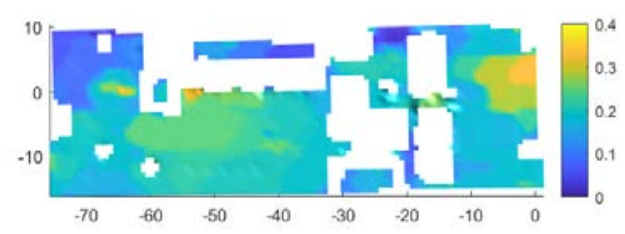

(a) 5 scan lines

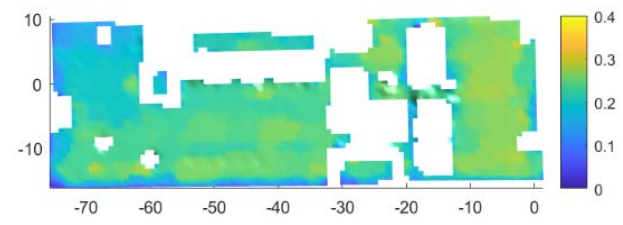

(c) 15 scan lines

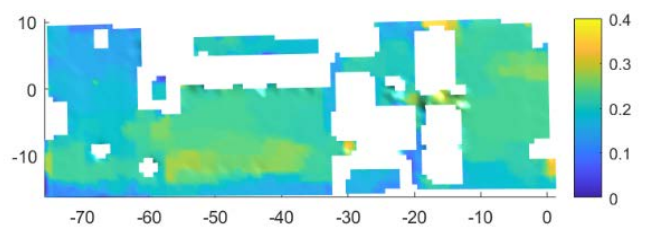

(b) 10 scan lines

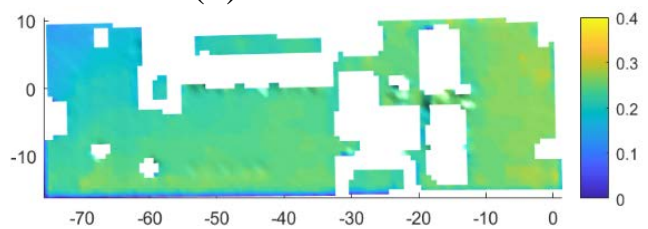

(d) 20 scan lines

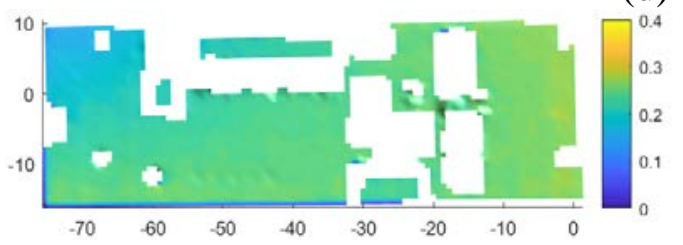

(e) 25 scan lines

Fig. 11. Recovered displacement images (mm) with different number of scan lines.

Image recovery by $\ell_{1}$ optimisation, in the case of 25 scan lines, takes around 20 seconds on a standard desk-top computer. For the full series of 3158 images the recovery time amounts to an excessive 18 hours. As has already been suggested, one way to overcome this problem is to carry out a preliminary SD reduction. Fig. 12 shows the correlation functions for the first eight SD kernel functions using the same 9 scan-lines as in Fig. 6. Clearly the kernel functions are sparser than the raw DIC data. Whereas the average correlation coefficient for the kernels is over $88 \%$, that for the displacement images is only around $65 \%$. In general the sparsity 
reduces as the index of the kernels increases, which is the likely reason for the fall in the correlation coefficient for the eighth kernel shown in the figure.

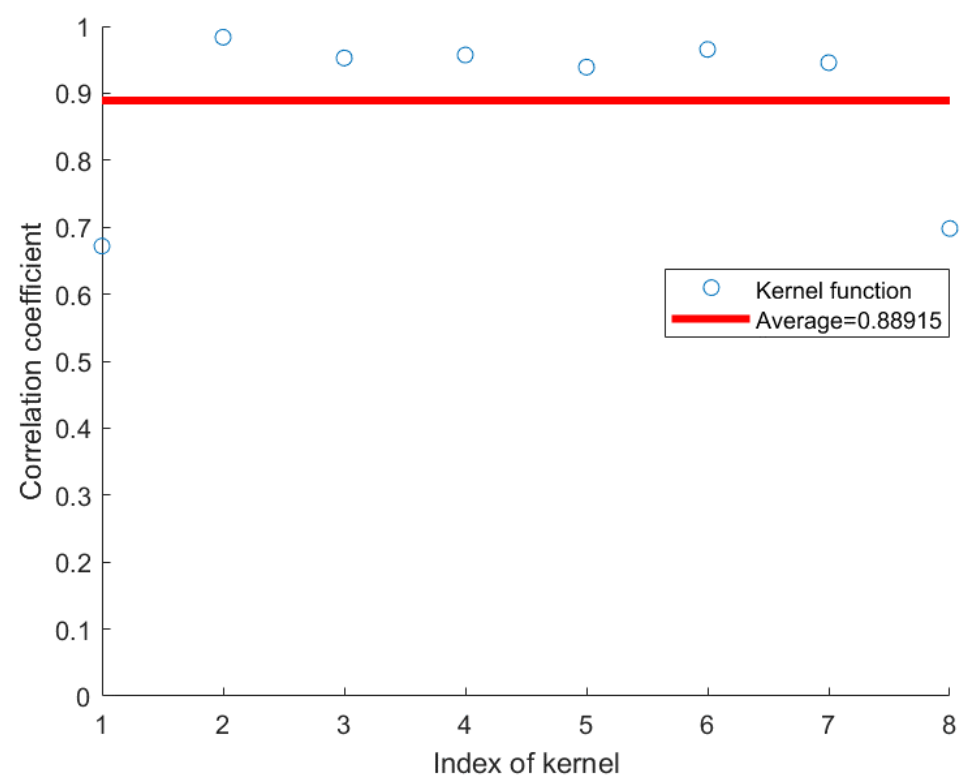

Fig. 12. Correlation coefficients for kernel functions of circuit board part 2.

\section{Compressed Sensing for OMA}

Following the CS recovery, operational modal analysis of the SD data was carried out according to the procedures described in §2 (and in [37]). Modal identification requires only the signals in the SD domain, then mode-shape visualisation can be achieved using the kernel functions. In the present example, the determination of modal properties was conducted using the stochastic subspace identification (SSI) algorithm [41]. By using the SD technique described in $\S 6$, there were found to be eight significant kernel function terms in the updated basis; the identified frequencies from [38] are presented in Table 1.

Table 1. Identified natural frequencies from the updated basis of the circuit board.

\begin{tabular}{|l|l|l|}
\hline Mode & 1 & 2 \\
\hline Frequency $(\mathrm{Hz})$ & 535.297 & 779.039 \\
\hline
\end{tabular}

Fig. 13 shows the number of uniformly distributed random samples of the SD signals and the number of radial scan lines of the kernels needed to exceed a threshold of 0.9 on the obtained MAC values, between the recovered and original SD data. It is clear that 650 random samples and 9 scan lines are sufficient. The resulting mode shapes are shown in Fig. 14 where close agreement between the recovered and original SD images can be observed. Of course, an even greater number of random samples and scan lines would result in even closer agreement. 


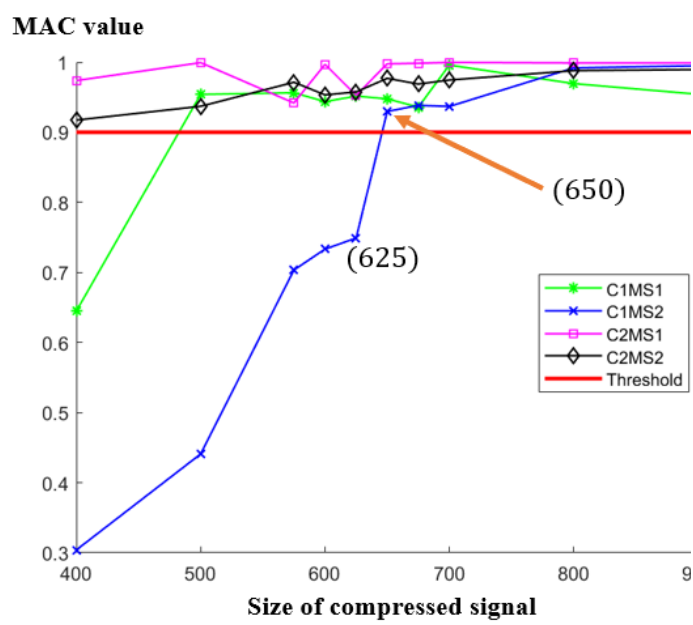

(a) Threshold for SD signals.

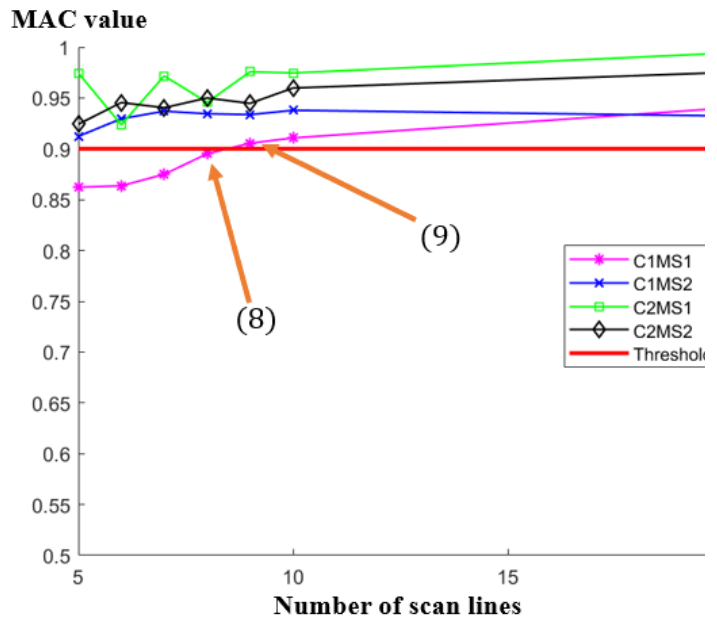

(b) Threshold for kernel functions.

Fig. 13. Sampling requirements to exceed MAC threshold of 0.9 (C - circuit board, MS mode shape).

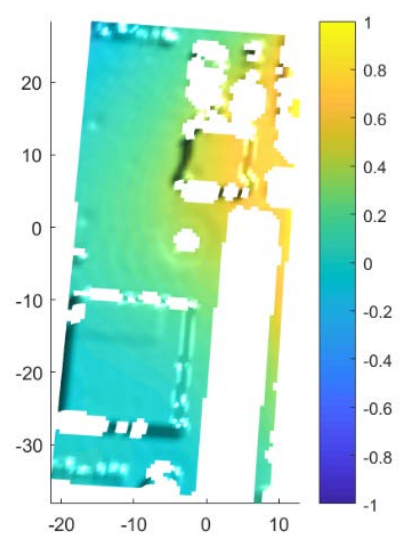

(a) $1^{\text {st }}$ mode shape of circuit board part 1 from original SD data

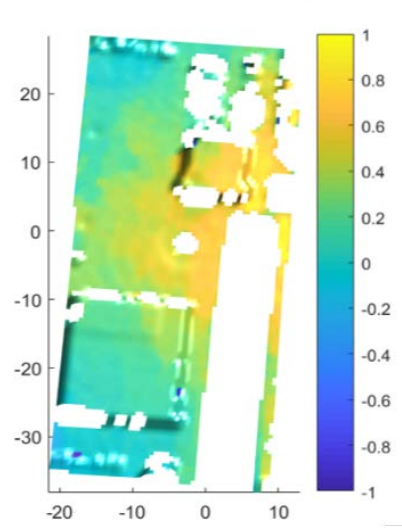

(c) $1^{\text {st }}$ mode shape of circuit board part 1 from recovered SD data

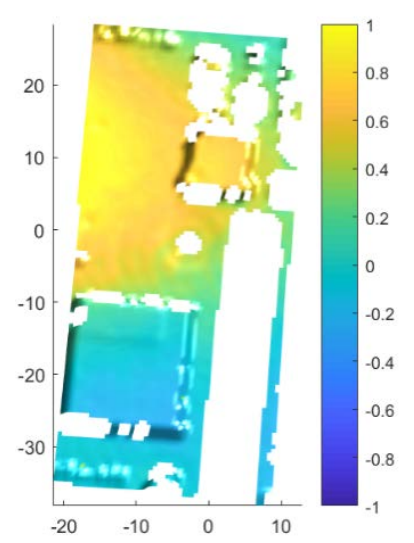

(b) $2^{\text {nd }}$ mode shape of circuit board part 1 from original SD data

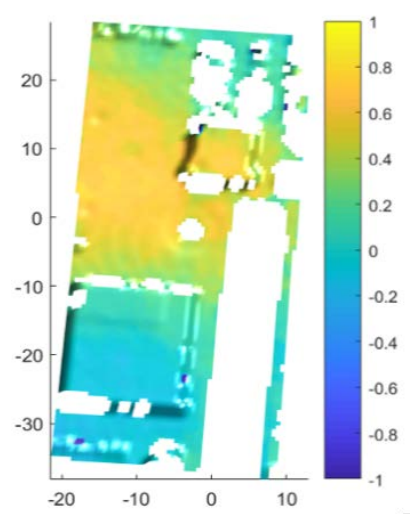

(d) $2^{\text {nd }}$ mode shape of circuit board part 1 from recovered SD data 


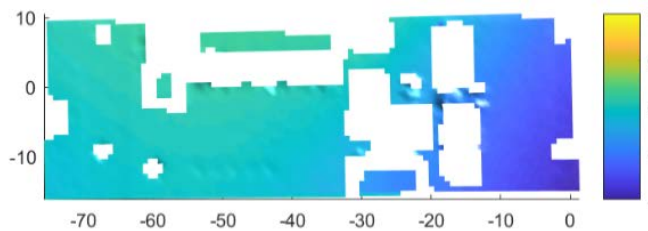

(e) $1^{\text {st }}$ mode shape of circuit board part 2 from original SD data

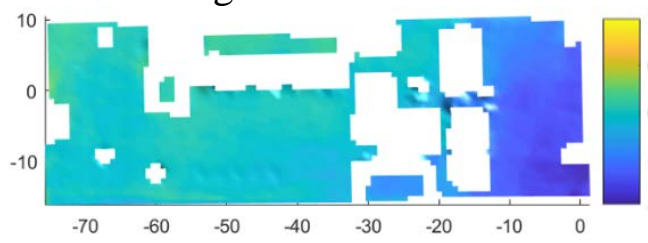

(g) $1^{\text {st }}$ mode shape of circuit board part 2 from recovered SD data

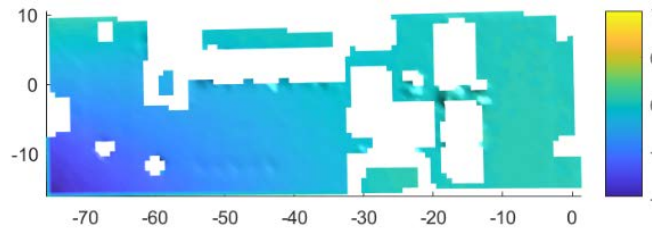

(f) $2^{\text {nd }}$ mode shape of circuit board part 2 from original SD data

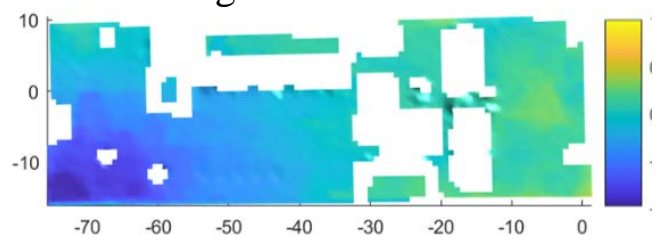

(h) $2^{\text {nd }}$ mode shape of circuit board part 2 from recovered SD data

Fig. 14. The comparison of identified mode shapes of data measured from circuit board.

Natural frequencies from original and recovered SD data are presented in Table 2, where differences are given in percentage terms. The compression of signals and kernel functions achieved by CS over prior-reduced SD data is given in Tables 3 and 4 for parts 1 and 2 of the circuit board respectively. It is seen that the effect of CS is the reduction of kernels by more than fourteen and eleven times for the two parts. In the authors earlier work [38] compression ratios of over 160 and 110 for parts 1 and 2 were obtained by SD basis updating. When multiplied by the present compression ratios the data needed for storage and transmission is reduced by more than 1300 times for part 1 and 720 times for part 2 .

Also from Tables 3 and 4 it is seen that the compression ratio for SD signals is close to 5 . It is clear the second approach postulated in $\S 4$, of compressing the 8091 DIC date-point signals, is likely to be highly inefficient when compared to the proposed approach, even if the compressive ratio of 5 were to be exceeded (doubled or greater) for at least some of the datapoint signals.

Table 2. Identified natural frequencies $(\mathrm{Hz})$

\begin{tabular}{|l|l|l|}
\hline Mode & 1 & 2 \\
\hline Original SD data & 535.297 & 779.039 \\
\hline Recovered SD data & 533.238 & 779.740 \\
\hline Difference in \% & 0.385 & 0.090 \\
\hline
\end{tabular}

Table 3. Compression ratios for signals and kernel functions: circuit board part 1 .

\begin{tabular}{|l|l|l|l|l|}
\hline & Signal size & \multirow{2}{*}{ Compression ratio } & Kernel & \multirow{2}{*}{ Compression ratio } \\
\cline { 1 - 3 } Original & & & \\
\cline { 1 - 2 } proposed method & 658 time instants & \multirow{2}{*}{4.86} & 5300 spatial data & \multirow{2}{*}{14.29} \\
& & 371 measurements & \\
\hline
\end{tabular}

Table 4. Compression ratios for signals and kernel functions: circuit board part 2.

\begin{tabular}{|l|l|l|l|l|}
\hline & Signal size & \multirow{2}{*}{ Compression ratio } & Kernel & \multirow{2}{*}{ Compression ratio } \\
\hline Original & & & \\
\cline { 1 - 4 } proposed method & 3158 time instants & \multirow{2}{*}{4.86} & 2791 spatial data & \multirow{2}{*}{11.3} \\
\cline { 3 - 4 } & & & 247 measurements & \\
\hline
\end{tabular}




\section{Conclusions}

In-situ data compression and remote reconstruction is an important practical issue for numerous industries that rely upon efficient data storage and transmission. However, CS alone is found to be prohibitively expensive for the processing of many thousands of camera images, each containing typically tens or hundreds of thousands of data points. A solution to this problem is presented in the form of a combined approach whereby CS is preceded by SD decomposition and applied to SD time signals and kernel functions. This procedure is demonstrated on industrial DIC data from a partially observed printed circuit board. Further significant compression is achieved (beyond the SD reduction) and OMA is carried out successfully on CS-recovered data. As described in the Introduction, CS techniques are in widespread use across numerous disciplines. Therefore the potential value of the combined SD/CS methodology extends beyond the testing and monitoring of engineering structures, wherever large 2D or 3D images must be reduced for reasons of efficiency and speed of reconstruction.

\section{Acknowledgement}

Yen-Hao Chang wishes to acknowledge the support provided by a University of Liverpool / NTHU scholarship through the dual PhD programme. DIC data from the printed circuit board were kindly provided by Dr Thorsten Siebert of Dantec Dynamics GmbH.

\section{References}

1. F. Ubertini, G. Comanducci, N. Cavalagli. Vibration-based structural health monitoring of a historic belltower using output-only measurements and multivariate statistical analysis, Structural Health Monitoring 15 (4) (2016) 438-457.

2. Weijtjens, W.; Verbelen, T.; De Sitter, G.; Devriendt, C. Foundation structural health monitoring of an offshore wind turbine-A fullscale case study, Structural Health Monitoreing 15 (4) (2015) 389-402.

3. G. Hackmann, W. Guo, G. Yan, Z. Sun, C. Lu, S. Dyke, Cyber-physical codesign of distributed structural health monitoring with wireless sensor networks, IEEE Trans. Parallel Distributed Systems 25 (1) (2014) 63-72.

4. M. Y. Bhuiyan, J. Bao, B. Poddar, V. Giurgiutiu. Toward identifying crack-length-related resonances in acoustic emission waveforms for structural health monitoring applications. Structural Health Monitoring 17(3) (2017) 577-585.

5. Feng, D., \& Feng, M. Q. Experimental validation of cost-effective vision-based structural health monitoring. Mechanical Systems and Signal Processing 88 (2017) 199-211.

6. T. Khuc and F. N. Catbas, Completely contactless structural health monitoring of real-life structures using cameras and computer vision, Structural Control and Health Monitoring 24 (1) (2016) e1852.

7. Y. Yang, C. Dorn, T. Mancini, Z. Talken, G. Kenyon, C. Farrar, D. Mascareñas. Spatiotemporal video-domain high-fidelity simulation and realistic visualization of full-field dynamic responses of structures by a combination of high-spatial-resolution modal model and video motion manipulations, Structural Control and Health Monitoring 25 (8) (2018) e2193.

8. N. Wadhwa, M. Rubinstein, F. Durand, W. T. Freeman. Phase-based video motion processing. ACM Transactions on Graphics 32 (4) (2013) 80.1-9.

9. Y. Yang, S. Nagarajaiah. Robust data transmission and recovery of images by compressed sensing for structural health diagnosis. Structural Control and Health Monitoring 24 (1) (2017) e1856.

10. D. Donoho, Compressed sensing, IEEE Trans. Information Theory 52 (4) (2006) 1289-1306.

11. Cawley, P. Structural health monitoring: Closing the gap between research and industrial deployment. Structural Health Monitoring 17 (5) (2018) 1225-1244.

12. J. P. Amezquita-Sanchez, H. Adeli. Signal Processing Techniques for Vibration-Based Health Monitoring of Smart Structures. Archives of Computational Methods in Engineering, 23 (1) (2016) 1-15.

13. L. Yu, B. Pan. Color Stereo-Digital Image Correlation Method Using a Single 3CCD Color Camera. Experimental Mechanics, 51 (4) (2017) 649-657.

14. L. Yu, B. Pan. Single-camera high-speed stereo-digital image correlation for full-field vibration measurement. Mechanical Systems and Signal Processing 94 (2017) 374-383.

15. L. Yu, B. Pan. Full-frame, high-speed 3D shape and deformation measurements using stereo-digital image correlation and a single color high-speed camera. Optics and Lasers in Engineering 95 (2017) 17-25.

16. Y. Chi, L. Yu, B. Pan. Low-cost, portable, robust and high-resolution single-camera stereo-DIC system and its application in hightemperature deformation measurements. Optics and Lasers in Engineering. 104 (2018) 141-148.

17. A.J. Molina-Viedma, L. Felipe-Sesé, E. López-Alba, F.A. Díaz, High frequency mode shapes characterization using Digital Image Correlation and phasebased motion magnification. Mechanical Systems and Signal Processing 102 (2018) $245-261$.

18. K. Patil, V. Srivastava, J. Baqersad, A multi-view optical technique to obtain mode shapes of structures. Measurement 122 (2018) 358-367.

19. E. Candès, J. Romberg, and T. Tao. Robust uncertainty principles: Exact signal reconstruction from highly incomplete frequency information. IEEE Trans. Information Theory. 52 (2) (2006) 489-509.

20. M. Lustig, D. L. Donoho, J. M. Santos, and J. M. Pauly. Compressed sensing MRI. IEEE Signal Processing Magazine 25 (2) (2008), 72-82. 
21. J. W. Sanders, H. Song, S. J. Frank, T. Bathala, A. M. Venkatesan, Mitchell Anscher, C. Tang, T. L. Bruno, W. Wei, J. Ma. “Parallel imaging compressed sensing for accelerated imaging and improved signal-to-noise ratio in MRI-based postimplant dosimetry of prostate brachytherapy. Brachytherapy 17 (5) (2018) 816-824.

22. J. H. Yoon, S. M. Lee, H.-J. Kang, E. Weiland, E. Raithel, Y. Son, B. Kiefer, J. M. Lee. Clinical feasibility of 3-dimensional magnetic resonance cholangiopancreatography using compressed sensing: comparison of image quality and diagnostic performance Investigative Radiology 52 (10) (2017) 612-619.

23. Z. Lai, X. Qu, Y. Liu, D. Guo, J. Ye, Z. Zhan, Z. Chen. Image reconstruction of compressed sensing MRI using graph-based redundant wavelet transform. Medical Image Analysis 27 (2016) 93-104.

24. S. Arridge, P. Beard, M. Betcke, B. Cox, N. Huynh, F. Lucka, O. Ogunlade, E. Zhang. Accelerated high-resolution photoacoustic tomography via compressed sensing. Physics in Medicine and Biology 61 (2016) 8908-8940.

25. D. Gangopadhyay, E. G. Allstot, A. M. R. Dixon, K. Natarajan, S. Gupta, D. J. Allstot. Compressed Sensing Analog Front-End for Bio-Sensor Applications. IEEE Journal of Solid-State Circuits, 49 (2) (2016) 426-438.

26. D. Craven, B. McGinley, L. Kilmartin, M. Glavin, and E. Jones, “Compressed sensing for bioelectric signals: a review. IEEE Journal of Biomedical and Health Informatics 19 (2) (2015) 529-540.

27. S. Felix, R. Bolzern, and M. Battaglia, A compressed sensing-based image reconstruction algorithm for solar flare x-ray observations. The Astrophysical Journal 849 (1) (2017) 10pp.

28. C. S. Oxvig, T. Arildsen, and T. Larsen, Structure assisted compressed sensing reconstruction of undersampled AFM images. Ultramicroscopy 172 (2017) 1-9.

29. Y. Yang, S. Nagarajaiah. Output-only modal identification by compressed sensing: Non-uniform low-rate random sampling Mechanical Systems and Signal Processing 56-57 (2015) 15-34.

30. S. Qin, J. Guo and C Zhu, Sparse component analysis using time-frequency representations for operational modal analysis Sensors 15 (2015) 6497-6519.

31. J.Y. Park, M.B. Wakin and A.C. Gilbert. Modal analysis with compressive measurement. IEEE Transactions on Signal Processing 62(7) (2014) 1655-1670.

32. M.K. Hu. Visual pattern recognition by moment invariants. IRE Transactions on Information Theory IT-8, (1962) 179-187.

33. W. Wang, J.E. Mottershead and C. Mares. Mode-shape recognition and finite element model updating using the Zernike moment descriptor. Mechanical Systems and Signal Processing 23(7) (2009) 2088-2112.

34. W. Wang, J.E. Mottershead, A. Ihle, T. Siebert, H.R. Schubach. Finite element model updating from full-field vibration measurement using digital image correlation. Journal of Sound and Vibration 330(8) (2011) 1599-1620.

35. W. Wang, J.E. Mottershead, C.M. Sebastian and E.A. Patterson. Shape features and finite element model updating from full-field strain data. International Journal of Solids and Structures 48(11-12) (2011) 1644-1657.

36. W. Wang, J. Mottershead, T. Siebert, A. Pipino Frequency response functions of shape features from full-field vibration measurements using digital image correlation Mechanical. Systems and Signal Processing 28 (2012) 333-347.

37. Y.-H. Chang, W. Wang, T. Siebert, J.-Y. Chang, J. E. Mottershead. Basis-updating for data compression of displacement maps from dynamic DIC measurements. Mechanical Systems and Signal Processing 115 (2019) 405-417.

38. M. Aharon, M. Elad, A. M. Bruckstein. The K-SVD: an algorithm for designing of overcomplete dictionaries for sparse representation, IEEE Transactions on Signal Processing 54 (2006) 4311-4322.

39. E. J. Candes and M. B. Wakin, An introduction to compressive sampling. IEEE Signal Processing Magazine, 25 (2) (2008) 21-30.

40. E. Cande`s and J. Romberg, $\ell 1$-MAGIC: Recovery of sparse signals via convex programming, California Inst. Technol., Pasadena, CA, Tech. Rep., Oct. 2005.

41. B. Peeters, G. De Roeck, Referenced-based stochastic subspace identification for output-only modal analysis. Mechanical Systems and Signal Processing 13 (6) (1999) 855-878. 